\title{
Life possible in the early Universe
}

\section{Planets orbiting the first stars could have been habitable, challenging arguments for a multiverse.}

\section{BY ZEEYA MERALI}

A liens might have existed during the Universe's infancy. A set of calculations suggests that liquid water - a prerequisite for life - could have formed on rocky planets just 15 million years after the Big Bang.

Abraham Loeb, an astrophysicist at Harvard University in Cambridge, Massachusetts, has realized that in the early Universe, the energy required to keep water liquid could have come from the cosmic microwave background, the afterglow of the Big Bang, rather than from host stars. Today, the temperature of this relic radiation is just 2.7 kelvin, but at an age of around 15 million years it would have kept the entire Universe at a balmy 300 kelvin, says Loeb, who posted his calculations to the arXiv preprint server this month (http://arxiv.org/ abs/1312.0613).

Loeb says that rocky planets could have existed at that time, in pockets of the Universe where matter was exceptionally dense, leading to the formation of massive, short-lived stars that would have enriched these pockets in the heavier elements needed to make planets. He suggests that

"The whole Universe was once an incubator for life." there would have been a habitable epoch of 2 million or 3 million years during which all rocky planets would have been able to maintain liquid water, regardless of their distance from a star. "The whole Universe was once an incubator for life," he says.

Loeb's result also challenges the anthropic principle, a line of reasoning that is invoked to explain why certain physical parameters seem to be tuned to the precise values needed for life: the Universe is the way it is because beings exist to observe it. The principle is consistent with the idea of a multiverse: if multiple universes exist, each based on different parameters, then intelligent beings should not be surprised to find themselves in one in which those parameters are suited to life.

In the 1980s, Nobel laureate and physicist Steven Weinberg used an anthropic argument to calculate a maximum value for a measure of the intrinsic energy of the vacuum in space that, in theory, would push space outwards. Weinberg pointed out that unless this value is tiny, it would have torn matter apart before the Sun, Earth or humans could have come into existence. His prediction seemed to be confirmed in the late 1990s, when astronomers discovered dark energy, which seems to act like a vacuum force that accelerates the expansion of the Universe - but by only a small amount. According to particle physicists' calculations, dark energy should actually be some 120 orders of magnitude stronger than Weinberg's maximum value. Multiverse proponents take this as evidence that multiple universes exist that have higher values of vacuum energy.

But during Loeb's proposed habitable epoch, matter was so dense that even if the vacuum energy had been a million times stronger, it would not have prevented the formation of stars and rocky planets, and the emergence of life. Thus, Loeb says, advocates of the anthropic principle cannot claim that the small value observed now is the only one that could be observed by living beings.

Responses to Loeb's work vary. Christopher Jarzynski, a biophysicist at the University of Maryland, College Park, is not convinced that life could exist in a uniformly warm Universe. Life on Earth depends thermodynamically not only on the heat source of the Sun, but also on the cold cosmic microwave background, which provides a heat sink, he notes. "Life feeds off this," he says. And Alexander Vilenkin, a cosmologist at Tufts University in Medford, Massachusetts, says that a few million years is too short a time to produce intelligent life.

Yet Freeman Dyson, a physicist at the Institute for Advanced Study in Princeton, New Jersey, thinks that life might be more adaptable than we think. "Anything is habitable if you are clever enough," he says. -

\section{CORRECTION}

The News story 'Image search triggers Italian police probe' (Nature 504, 18; 2013) should have said that paper identified as problematic and being retracted was in Cell Death and Differentiation, not Cell Death and Disease. 